\title{
PREDIÇÃO E CORRELAÇÃO DE PROPRIEDADES TERMODINÂMICAS DO ÁCIDO ACÉTICO APLICANDO EQUAÇÕES DE ESTADO
}

\author{
M.K.S. MONTEIRO ${ }^{1}$, I.F.R. BRUNELLI ${ }^{2}$ e P.J.M. LIMA ${ }^{3}$ \\ ${ }^{1}$ Universidade Federal do Rio Grande do Norte, Programa de Pós-Graduação em Engenharia Química \\ ${ }^{2}$ Universidade Federal de São Carlos, Departamento de Engenharia Química \\ ${ }^{3}$ Universidade Federal Rural do Semi-Árido, Departamento de Engenharia Química \\ E-mail para contato: mayra.kerolly@gmail.com
}

\begin{abstract}
RESUMO - O presente trabalho tem como objetivo realizar o estudo comparativo entre a modelagem matemática e os dados experimentais das propriedades termodinâmicas do ácido acético $\left(\mathrm{CH}_{3} \mathrm{COOH}\right)$ puro utilizando equações de estado cúbicas (EDEC) como a de Peng-Robinson (PR) e a de Soave-Redlich-Kwong com associação a equação de estado Cubic-Plus-Association (SRK+CPA) para a descrição do comportamento PVT desta substância, de modo a verificar se estas equações representam de forma aceitável a realidade. Os dados experimentais foram obtidos em artigos científicos e através da base de dados do DIPPR (Design Institute for Physicol Properties). Para a predição dos dados termodinâmicos do ácido acético, utilizou-se a EDEC PR através do software Peng-Robinson.exe (PR) e a EDEC SRK+CPA através do software SPECS (versão 5.63). A partir da análise estatística, foi evidenciado que o modelo termodinâmico SRK+CPA foi a EDEC que melhor se ajustou ao comportamento PVT do ácido acético em relação à correlação estabelecida com a EDEC de PR, sendo então a SRK+CPA a EDEC que melhor prediz as propriedades termodinâmicas do ácido acético.
\end{abstract}

\section{INTRODUÇÃO}

Propriedades termodinâmicas de substâncias puras e de misturas e dados de Equilíbrio Líquido-Vapor (ELV) são de fundamental importância para a indústria química, pois são parâmetros básicos para o projeto de equipamentos, como a torre de destilação, por exemplo, otimização de processos, desenho de plantas químicas, dentre outras etapas de desenvolvimento de projetos da engenharia química (CARMO, 2009).

A predição das propriedades termodinâmicas e de equilíbrio de fases de substâncias puras e de misturas multicomponente continua sendo um grande desafio no meio científico, ao mesmo tempo em que se torna uma necessidade no meio industrial. Com o crescente uso de recursos computacionais e softwares de simulação de processos, o desenvolvimento de modelos termodinâmicos foi grandemente impulsionado e estes modelos devem ter características como simplicidade, flexibilidade, generalidade, confiabilidade e precisão (STAUDT, 2010).

Para prever o comportamento termodinâmico e cálculo de ELV utiliza-se de equações de estado que definem o estado físico-químico da matéria, sendo ao longo dos anos muitas dessas equações desenvolvidas, cada uma com características específicas no que diz respeito à base teórica, complexidade matemática e potencial de aplicação. 
Neste trabalho, serão utilizadas especificamente as equações de estado cúbicas PR e SRK+CPA. Fazendo uso das equações mencionadas, e diante da importância de se conhecer as propriedades termodinâmicas das substâncias, a fim de dimensionar e prever o comportamento de um processo, este trabalho teve como objetivo aplicar as referidas equações de estado para correlacionar e predizer o comportamento de propriedades termodinâmicas do ácido acético.

\section{METODOLOGIA}

O comportamento PVT do ácido acético será correlacionado com a predição dos modelos termodinâmicos para verificar qual equação cúbica é capaz de descrever tal comportamento da substancia pura sem a necessidade da realização de experimentos em laboratório.

Para a predição das propriedades foi utilizado dois simuladores o software PengRobinson.exe (PR), aplicando a equação de estado de Peng-Robinson e o software SPECS (v5.63), no qual aplica a equação de estado cúbica de Soave-Redlich-Kwong com associação. Os softwares MS Excel e Origin 8 também foram utilizados para apresentar os dados simulados através de gráficos. Os dados experimentais do ácido acético empregados para validação dos modelos foram obtidos em artigo disposto no programa DIPPR.

A análise do comportamento PVT foi realizada através dos diagramas PV e HP utilizando os desvios absolutos e relativos, calculados entre os valores experimentais dispostos na literatura e os simulados pelos softwares, conforme a equação 1. Por meio destes desvios foi permitido quantificar o ajuste do modelo em relação aos dados obtidos experimentalmente.

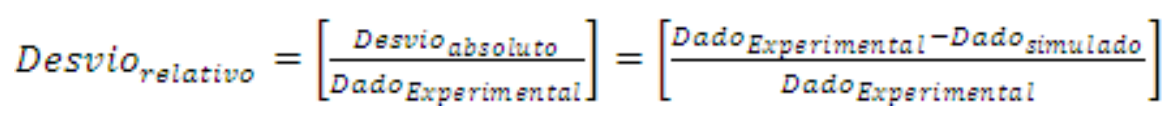

\subsection{Diagrama PV}

Foi utilizado o simulador PR, para a construção do diagrama PV. Com isso, na faixa de temperatura compreendida entre o ponto de bolha $(391,1 \mathrm{~K})$ e o ponto crítico $(592,7 \mathrm{~K})$ do ácido acético com o passo de $6.5 \mathrm{~K}$, a equação de Peng-Robinson prediz os dados referentes às isotermas de líquido sub-resfriado e vapor superaquecido a partir do ponto de saturação 28,5 bar e $540 \mathrm{~K}$, onde a pressão para líquido sub-resfriado foi variada entre 28,5 e 78,5 bar, já para o vapor superaquecido sua pressão foi variada entre 1 e 28,5 bar. As isotermas crítica e supercrítica, por sua vez, foram geradas com temperaturas de 592,7 e $610 \mathrm{~K}$, respectivamente. As propriedades intrínsecas do ácido acético estão apresentadas na Tabela 01.

Tabela 01 - Propriedades intrínsecas do ácido acético

\begin{tabular}{|c|c|c|c|}
\hline Substância: & Ácido Acético & Temperatura Crítica (K): & 592,7 \\
\hline Fórmula: & $\mathrm{CH}_{3} \mathrm{COOH}$ & Pressão Crítica (bar): & 57,9 \\
\hline $\begin{array}{c}\text { Peso Molecular } \\
\text { (g/mol): }\end{array}$ & 60,052 & Volume Crítico $\left(\mathbf{c m}^{\mathbf{3}} \mathbf{m} \mathbf{m o l}\right):$ & 171 \\
\hline $\begin{array}{c}\text { Temperatura no } \\
\text { Ponto de Bolha (K): }\end{array}$ & 393,1 & $\begin{array}{c}\text { Fator de } \\
\text { compressibilidade }(\boldsymbol{\omega}):\end{array}$ & 0,201 \\
\hline
\end{tabular}

Fonte: Autoria Própria 


\subsection{Diagrama HP}

Para a construção do diagrama HP, utilizou-se tanto o programa PR quanto o SPECS v5.63 para calcular a entalpia de vaporização a temperatura constante de $391.1 \mathrm{~K}$, numa faixa de pressão compreendida entre 1 e 10 bar.

\section{RESULTADOS E DISCUSSÃO}

\subsection{Diagrama PV}

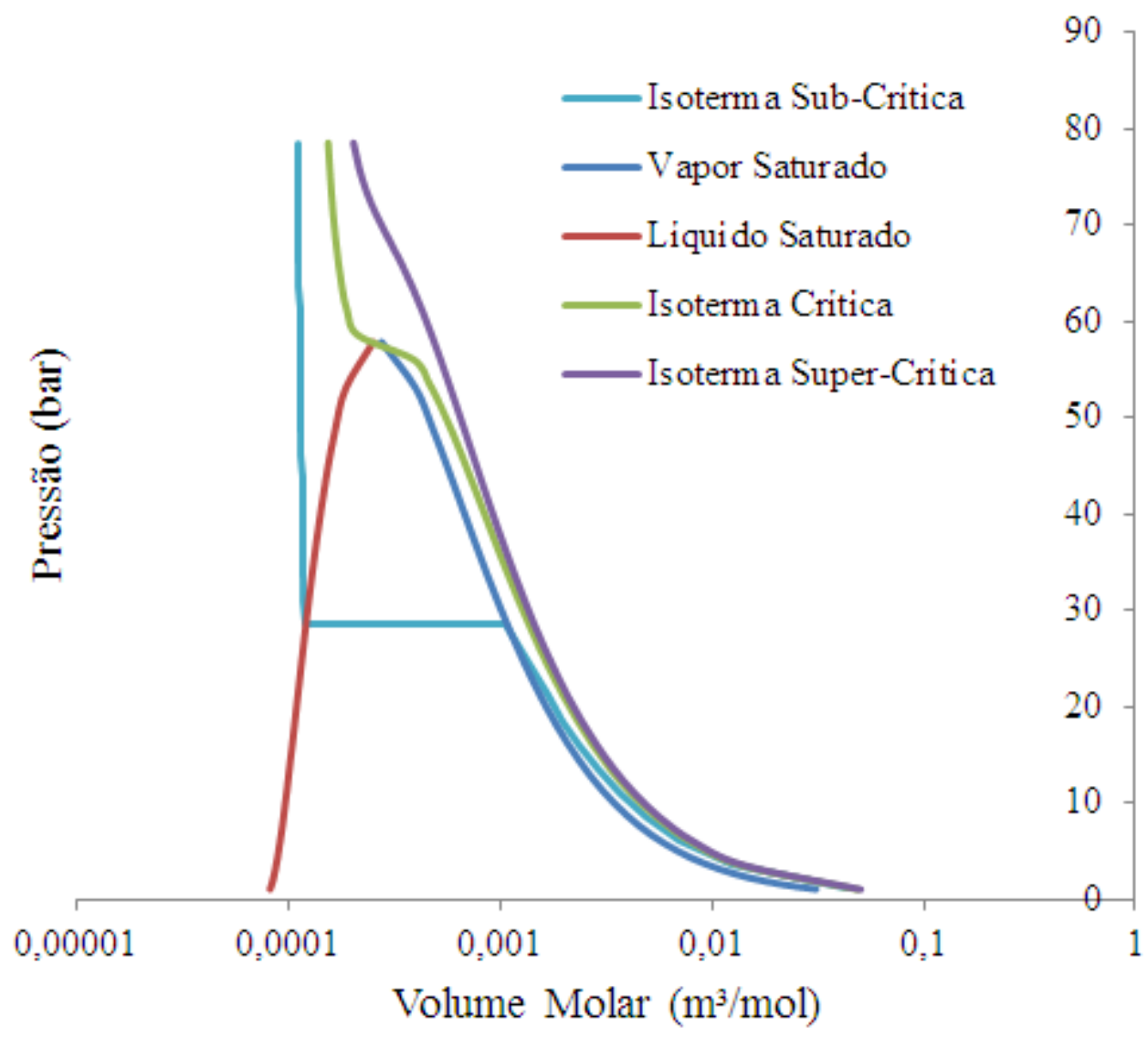

Fonte: Autoria Própria

Figura 01 - Diagrama de Pressão versus Volume Molar

A Fig. 01 mostra as regiões do líquido, líquido/vapor e vapor em um diagrama PV, com a adição de três isotermas. Abaixo da curva em forma de domo encontra-se a região bifásica líquido/vapor, a metade representada pela curva de cor vermelha identifica a curva do líquido saturado, onde a esquerda dessa curva encontra-se a região do líquido sub-resfriado e a outra metade do domo representado pela curva azul indica a curva de vapor saturado, e a direita dessa curva encontra-se a região do vapor superaquecido. As três isotermas traçadas foram: isoterma sub-crítica $\left(T<T_{c}\right)$, isoterma crítica $\left(T=T_{c}\right)$ e isoterma supercrítica $\left(T>T_{c}\right)$. $O$ segmento horizontal da isoterma sub-crítica representa todas as misturas possíveis de líquido 
e vapor em equilíbrio, por isso encontra-se dentro da curva em forma de domo. A isoterma crítica exibe uma inflexão horizontal no ponto crítico no topo do domo, onde as fases líquidas e vapor se tornam indistinguíveis. Isotermas na região do líquido sub-resfriado são bastante inclinadas, porque o volume de líquido varia pouco com grandes variações nas pressões.

No intuito de fazer a correlação do comportamento dos dados experimentais, fornecido por LAGO et al. 2009, com o comportamento predito pelo software PR baseado no modelo Peng-Robinson, foram calculados os erros relativos para se observar o quão próximo do valor real os dados experimentais estão e para qual isoterma obteve-se o menor erro médio. É relevante observar que os menores erros ocorreram para temperaturas maiores e menores pressões. Através da análise, pode-se concluir que os erros dos dados experimentais em relação ao teórico da equação de Peng-Robinson são altos, entorno de $10 \%$, o que põe em questão a confiabilidade dos dados experimentais ou a necessidade de correlacionar as propriedades termodinâmicas do ácido acético com outro modelo termodinâmico.

\subsection{Diagrama HP}

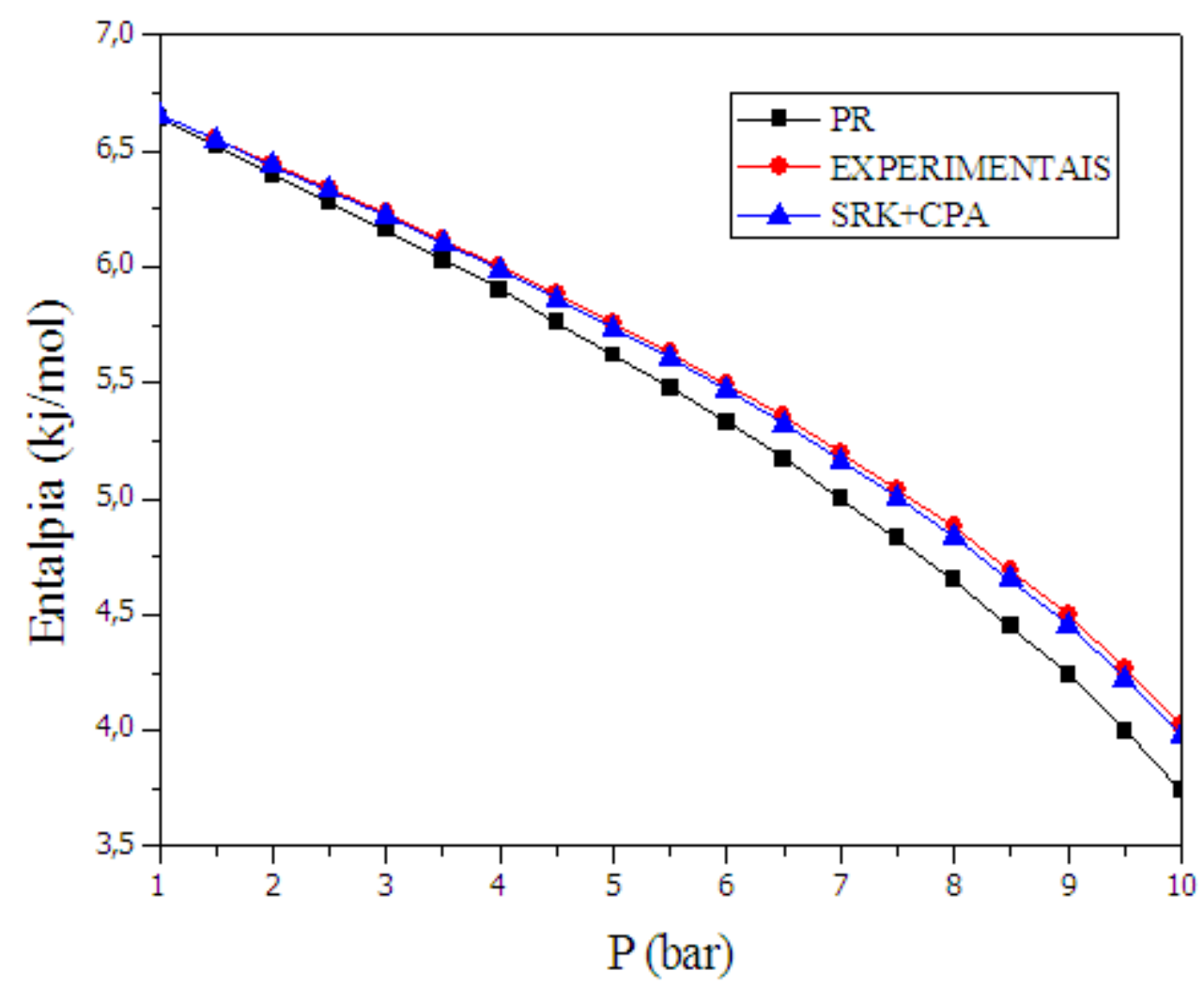

Figura 02 - Diagrama da entalpia versus pressão 
Tabela 02 - Desvios dos dados experimentais relativos aos dados do simulador PR e SPECS.

\begin{tabular}{|c|c|c|c|c|c|}
\cline { 2 - 6 } \multicolumn{1}{c|}{} & $\begin{array}{l}\text { Simulador } \\
\text { PR }\end{array}$ & Experimental & $\begin{array}{l}\text { Simulador } \\
\text { SPECS }\end{array}$ & $\begin{array}{l}\text { Desvio } \\
\text { PR }\end{array}$ & $\begin{array}{l}\text { Desvio } \\
\text { SPECS }\end{array}$ \\
\hline P (bar) & \multicolumn{3}{|c|}{ Entalpia (kj/mol) } & \multicolumn{2}{c|}{} \\
\hline 1 & 6,64 & 6,655 & 6,6525 & $0,23 \%$ & $0,04 \%$ \\
\hline 1,5 & 6,52 & 6,55 & 6,545 & $0,46 \%$ & $0,08 \%$ \\
\hline 2 & 6,4 & 6,445 & 6,4375 & $0,70 \%$ & $0,12 \%$ \\
\hline 2,5 & 6,28 & 6,34 & 6,33 & $0,95 \%$ & $0,16 \%$ \\
\hline 3 & 6,16 & 6,235 & 6,2225 & $1,20 \%$ & $0,20 \%$ \\
\hline 3,5 & 6,03 & 6,12 & 6,105 & $1,47 \%$ & $0,25 \%$ \\
\hline 4 & 5,9 & 6,005 & 5,9875 & $1,75 \%$ & $0,29 \%$ \\
\hline 4,5 & 5,76 & 5,88 & 5,86 & $2,04 \%$ & $0,34 \%$ \\
\hline 5 & 5,62 & 5,755 & 5,7325 & $2,35 \%$ & $0,39 \%$ \\
\hline 5,5 & 5,48 & 5,63 & 5,605 & $2,66 \%$ & $0,44 \%$ \\
\hline 6 & 5,33 & 5,495 & 5,4675 & $3,00 \%$ & $0,50 \%$ \\
\hline 6,5 & 5,17 & 5,35 & 5,32 & $3,36 \%$ & $0,56 \%$ \\
\hline 7 & 5 & 5,195 & 5,1625 & $3,75 \%$ & $0,63 \%$ \\
\hline 7,5 & 4,83 & 5,04 & 5,005 & $4,17 \%$ & $0,69 \%$ \\
\hline 8 & 4,65 & 4,875 & 4,8375 & $4,62 \%$ & $0,77 \%$ \\
\hline 8,5 & 4,45 & 4,69 & 4,65 & $5,12 \%$ & $0,85 \%$ \\
\hline 9 & 4,24 & 4,495 & 4,4525 & $5,67 \%$ & $0,95 \%$ \\
\hline 9,5 & 4 & 4,27 & 4,225 & $6,32 \%$ & $1,05 \%$ \\
\hline 10 & 3,74 & 4,025 & 3,9775 & $7,08 \%$ & $1,18 \%$ \\
\hline & & & Desvio & $3,00 \%$ & $0,50 \%$ \\
\cline { 5 - 6 } & & & & & \\
\hline
\end{tabular}

Comparando então os dados experimentais estimados na faixa indicada por PIAZZA et al. 2011 com os dados preditos nas mesmas circunstâncias pelo modelo SRK+CPA e PengRobinson. Temos que para a equação de PR, o desvio médio relativo foi de 3,00\% e para o modelo de SRK+CPA esse valor foi de $0,50 \%$. Conquanto, observa-se que a equação que melhor prediz os valores de entalpia em função da pressão foi o modelo SRK+CPA, apresentando um menor desvio médio relativo aos dados experimentais, quando comparado ao desvio médio do modelo PR. Apesar de também não ter se mostrado o melhor, o modelo de Peng-Robinson apresentou um valor satisfatório de desvio médio relativo. Tal análise pode ser observada na Fig. 02, que mostra o comportamento da entalpia em função da pressão através dos dados da literatura e dos valores simulados a partir dos softwares empregados. Esse comportamento já era esperado, pois o modelo de Soave-Redlich-Kwong com associação propõe em seu equacionamento parâmetros associativos que consideram as interações desconsideradas pela equação de estado de Peng-Robinson, além de satisfazer a disposição macroscópica dos dados de entalpia a baixas temperaturas. Segundo ANDEREYA et al. (1990), o ácido acético não apresenta máximo da entalpia de vaporização em contraste com o seu fator de compressibilidade no vapor saturado. 


\section{CONCLUSÃO}

Com esse estudo foi possível concluir que as propriedades termodinâmicas do ácido acético foram preditas de maneira satisfatória tanto pela equação de estado cúbica de PengRobinson (PR), quanto pela equação de estado associada SRK+CPA, sendo a última a que melhor descreveu os dados obtidos experimentalmente na literatura, uma vez que apresentou o menor desvio médio nos casos analisados. Em linhas gerais, o trabalho possibilitou uma visão mais abrangente quanto à aplicação de modelos das equações de estado para predizer as propriedades termodinâmicas, além de permitir uma melhor compreensão do comportamento termodinâmico de uma substância pura, no ponto de vista teórico e computacional.

\section{REFERÊNCIAS}

ANDEREYA, E.; CHASE, J.D. chem. Eng. Technol. 13, p.304-312, 1990.

CARMO, F.B. DO. Desenvolvimento de ambiente computacional para cálculos termodinâmicos de substâncias puras e misturas e modelagem de Equilíbrio LíquidoVapor a pressões baixas e moderadas utilizando Equações de Estado. Monografia. Curso de Engenharia Química, UFC, Ceará, 2009.

LAGO, S.; ALBO, P.A.G. Thermodynamic properties of acetone calculated from accurate experimental speed of sound measurements at low temperatures and high pressures. $J$. Chem. Thermodynamics, 41, 506-512 (2009).

PIAZZA, L.; SPAN, R. An equation of state for acetic acid including the association term of saft. Fluid phase equilibria v. 303, p.134-149, 2011.

STAUDT, P. B, Nova equação cúbica de estado para a predição do equilíbrio liquid-vapor de misturas complexas envolvendo polímeros e óleos. 2010. 138 f. Tese de Doutorado Curso de Engenharia Química, UFRS, Rio Grande do Sul, 2010. 